PROCEEDINGS OF THE

AMERICAN MATHEMATICAL SOCIETY

Volume 133, Number 5, Pages 1267-1271

S 0002-9939(04)07826-8

Article electronically published on December 15, 2004

\title{
THE CONTENT OF A GAUSSIAN POLYNOMIAL IS INVERTIBLE
}

\author{
K. ALAN LOPER AND MOSHE ROITMAN
}

(Communicated by Bernd Ulrich)

\begin{abstract}
Let $R$ be an integral domain and let $f(X)$ be a nonzero polynomial in $R[X]$. The content of $f$ is the ideal $\mathfrak{c}(f)$ generated by the coefficients of $f$. The polynomial $f(X)$ is called Gaussian if $\mathfrak{c}(f g)=\mathfrak{c}(f) \mathfrak{c}(g)$ for all $g(X) \in R[X]$. It is well known that if $\mathfrak{c}(f)$ is an invertible ideal, then $f$ is Gaussian. In this note we prove the converse.
\end{abstract}

Let $R$ be a ring, that is, a commutative ring with unity. Let $A$ be a ring extension of $R$, and let $f(X) \in A[X]$ be a polynomial:

$$
f(X)=a_{n} X^{n}+\ldots+a_{1} X+a_{0} .
$$

The content ideal of $f$, designated by $\mathfrak{c}(f)=\mathfrak{c}_{R}(f)$, is the $R$-submodule of $A$ generated by the coefficients of $f$ in $A$. It is easy to see that if $f, g \in R[X]$ (and $A=R$ ), then $\mathfrak{c}(f g) \subseteq \mathfrak{c}(f) \mathfrak{c}(g)$. The inclusion in this statement is generally proper. The polynomial $f(X) \in R[X]$ is said to be Gaussian if $\mathfrak{c}(f g)=\mathfrak{c}(f) \mathfrak{c}(g)$ holds for all $g(X) \in R[X]$.

For general background on content ideals see [6]. There has been significant interest in recent years in the Dedekind-Mertens Lemma, which states that if $R$ is any commutative ring and $f, g \in R[X]$ are any two nonzero polynomials with $n$ being the degree of $g$, then $\mathfrak{c}(f g) \mathfrak{c}(f)^{n}=\mathfrak{c}(f)^{n+1} c(g)$. The polynomial $g$ is then Gaussian if for all polynomials $f$, the formula still holds when $n$ is replaced by zero. In [3] and [4] the more general question is examined concerning what the smallest integer is that can replace $n$ for a given polynomial $g$. In [2] a more combinatorial approach is taken to studying the Dedekind-Mertens Lemma with applications to ideals associated to content ideals (e.g. they examine primary decompositions of ideals of the form $\mathfrak{c}(f g))$. We note also that the Dedekind-Mertens Lemma implies that $\sqrt{\mathfrak{c}(f g)}=\sqrt{\mathfrak{c}(f) \mathfrak{c}(g)}$ for any polynomials $f(X), g(X)$ over an arbitrary ring $R$.

It is well known that if $\mathfrak{c}(f)$ is an invertible ideal of $R$, then $f$ is Gaussian. More generally, if $R$ is any ring and $\mathfrak{c}(f)$ is locally principal, then $f$ is Gaussian (see, e.g., [1, Theorem 1.1]). Recall that a nonzero ideal $I$ of an integral domain $R$ is

Received by the editors September 16, 2003.

2000 Mathematics Subject Classification. Primary 13B25.

Key words and phrases. Content, Gaussian polynomial, invertible ideal, locally principal, prestable ideal.

The second author thanks the Mathematics Department of Ohio State University for its hospitality.

(C)2004 American Mathematical Society Reverts to public domain 28 years from publication 
invertible iff it is locally principal, that is, iff $I R_{M}$ is a principal ideal for each maximal ideal $M$ of $R$.

It has been conjectured that the converse is true if $R$ is an integral domain (see [1] 7]), that is, a Gaussian polynomial over an integral domain has an invertible content. This question is included in the Ph.D. thesis of Kaplansky's student H. T. Tang. Significant progress has been made on this conjecture in two recent papers [7, 8]: in [7, Glaz and Vasconcelos prove the conjecture for $R$ integrally closed with some additional assumptions (including the Noetherian case). The general Noetherian case is settled in [8. As explained below, the conjecture has a local character; thus Heinzer and Huneke prove the conjecture for any locally Noetherian integral domain (this result is obtained as a particular case of a more general theorem).

The purpose of this note is to prove the conjecture for all integral domains. The Gaussian property of a polynomial $f(X) \in R[X]$ is local, that is, $f$ is Gaussian iff the image of $f$ in $R_{M}[X]$ is Gaussian for each maximal ideal $M$ of $R$. Thus to prove the conjecture we may assume that $R$ is quasilocal (cf. 7] and [8]). Moreover, this allows us to generalize the conjecture to the effect that if $R$ is locally an integral domain (that is, $R_{M}$ is a domain for each maximal ideal $M$ ), then a nonzero polynomial in $R[X]$ is Gaussian iff its content is locally principal (see Theorem 4 below).

Our approach is inspired by [7]. For a finitely generated ideal $I$ of $R$, let $\nu(I)$ be the minimal number of generators of $I$. To prove that $\mathfrak{c}(f)$ is invertible we first show that $\nu\left((\mathfrak{c}(f))^{n}\right)$ is bounded (Lemma 2 below), and conclude that $\mathfrak{c}_{R^{\prime}}(f)=\mathfrak{c}_{R}(f) R^{\prime}$ is invertible in $R^{\prime}$, the integral closure of $R$ (Lemma 3). To descend from $R^{\prime}$ to $R$ we simply "take conjugates" (see the proof of Theorem 4).

To bound the number of generators of $(\mathfrak{c}(f))^{n}$ we need the following proposition (actually, we use just the easier direction $[\Longleftarrow]$ ).

Proposition 1. Let $f(X)$ be a polynomial in $R[X]$ and let $n \geq 1$. Then $f(X)$ is Gaussian $\Longleftrightarrow f\left(X^{n}\right)$ is Gaussian.

Proof. Let $g$ by any polynomial in $R[X]$.

[ $\Longrightarrow$ Write

$$
g(X)=h_{0}\left(X^{n}\right)+X h_{1}\left(X^{n}\right)+\cdots+X^{n-1} h_{n-1}\left(X^{n}\right),
$$

where $h_{0}(X), \ldots, h_{n-1}(X)$ are polynomials in $R[X]$. Since $f$ is Gaussian, we obtain

$$
\begin{array}{r}
\mathfrak{c}\left(f\left(X^{n}\right)\right) \mathfrak{c}(g(X))=\mathfrak{c}\left(f\left(X^{n}\right)\right) \sum_{i=0}^{n-1} \mathfrak{c}\left(h_{i}\left(X^{n}\right)\right)=\sum_{i=0}^{n-1} \mathfrak{c}\left(f\left(X^{n}\right)\right) \mathfrak{c}\left(h_{i}\left(X^{n}\right)\right) \\
=\sum_{i=0}^{n-1} \mathfrak{c}(f(X)) \mathfrak{c}\left(h_{i}(X)\right)=\sum_{i=0}^{n-1} \mathfrak{c}\left(f(X) h_{i}(X)\right)=\sum_{i=0}^{n-1} \mathfrak{c}\left(f\left(X^{n}\right) X^{i} h_{i}\left(X^{n}\right)\right) \\
=\mathfrak{c}\left(f\left(X^{n}\right) \sum_{i=0}^{n-1} X^{i} h_{i}\left(X^{n}\right)\right)=\mathfrak{c}\left(f\left(X^{n}\right) g(X)\right) .
\end{array}
$$

Hence $f\left(X^{n}\right)$ is Gaussian.

$[\Longleftarrow]$ Since $f\left(X^{n}\right)$ is Gaussian, we obtain

$$
\mathfrak{c}(f(X) g(X))=\mathfrak{c}\left(f\left(X^{n}\right) g\left(X^{n}\right)\right)=\mathfrak{c}\left(f\left(X^{n}\right)\right) \mathfrak{c}\left(g\left(X^{n}\right)\right)=\mathfrak{c}(f(X)) \mathfrak{c}(g(X)) .
$$

Hence $f(X)$ is Gaussian. 
Lemma 2. Let $R$ be a quasilocal domain, let $f(X)$ be a Gaussian polynomial in $R[X]$, and let $I=\mathfrak{c}_{R}(f)$. Then

$$
\nu\left(I^{n}\right) \leq \operatorname{deg}(f)+1
$$

for sufficiently large $n \geq 1$.

Proof. By [5, Corollary 2] it is enough to show that $\nu\left(I^{2^{m}}\right) \leq \operatorname{deg}(f)+1$ for all $m \geq 0$. Let

$$
f(X)=g_{0}\left(X^{2}\right)+X g_{1}\left(X^{2}\right),
$$

where $g_{0}(X)$ and $g_{1}(X)$ are polynomials in $R[X]$. Since $\mathfrak{c}(f(-X))=\mathfrak{c}(f(X))$ and since $f(X)$ is Gaussian, we obtain

$$
\begin{aligned}
I^{2} & =(\mathfrak{c}(f))^{2}=\mathfrak{c}(f(X)) \mathfrak{c}(f(-X))=\mathfrak{c}(f(X) f(-X)) \\
& =\mathfrak{c}\left(g_{0}\left(X^{2}\right)^{2}-X^{2} g_{1}\left(X^{2}\right)^{2}\right)=\mathfrak{c}\left(g_{0}(X)^{2}-X g_{1}(X)^{2}\right) .
\end{aligned}
$$

Since $\operatorname{deg}\left(g_{0}(X)^{2}-X g_{1}(X)^{2}\right)=\operatorname{deg}(f)$, we infer that

$$
\nu\left(I^{2}\right) \leq \operatorname{deg} f+1 .
$$

Moreover, by Proposition [1, the polynomial $g_{0}(X)^{2}-X g_{1}(X)^{2}$ is Gaussian since $g_{0}\left(X^{2}\right)^{2}-X^{2} g_{1}\left(X^{2}\right)^{2}=f(X) f(-X)$ is a product of two Gaussian polynomials (to see that $f(-X)$ is Gaussian, put $-X$ for $X)$. Thus we may proceed by induction on $m$ to obtain $\nu\left(I^{2^{m}}\right) \leq \operatorname{deg} f+1$ for all $m \geq 0$. This concludes the proof of the lemma.

Lemma 3. Let $R$ be a quasilocal, integral domain, and let $f(X)$ be a Gaussian polynomial in $R[X]$. Then $\mathfrak{c}_{R^{\prime}}(f)=R^{\prime} \mathfrak{c}_{R}(f)$ is invertible in $R^{\prime}$.

Proof. By Lemma 2 $\nu\left(\mathfrak{c}_{R^{\prime}}\left(f^{n}\right)\right)$ is bounded. Hence, by [5] Corollary 1], the ideal $\mathfrak{c}_{R^{\prime}}(f)$ is prestable, and by [5] Lemma $\mathrm{F}$ ], it is an invertible ideal of $R^{\prime}$ (see also [7] Theorem 3.1]).

Theorem 4. Let $R$ be a ring which is locally a domain. Then a nonzero polynomial over $R$ is Gaussian iff its content in $R$ is locally principal.

Proof. We may assume that $R$ is quasilocal. Let $f(X)=\sum_{i=0}^{n} a_{i} X^{i}$ be a nonzero Gaussian polynomial in $R[X]$, and let $I=\mathfrak{c}_{R}(f)$. By the previous lemma, $I R^{\prime}$ is an invertible ideal in $R^{\prime}$. Let $1=\sum_{i=0}^{n} z_{i} a_{i}$, where $z_{i} \in\left(R^{\prime}: I\right)$ for all $i$. Let $g(X)=f(X) \sum_{i=0}^{n} z_{n-i} X^{i}=\left(\sum_{i=0}^{n} a_{i} X_{i}\right)\left(\sum_{i=0}^{n} z_{i} X^{n-i}\right)$. Thus $g(X)$ is a polynomial in $R^{\prime}[X]$, the coefficient of $X^{n}$ in $g(X)$ is 1 , and $f(X)$ divides $g(X)$ in $K[X]$, where $K$ is the fraction field of $R$ :

$$
g(X)=\sum_{i=0}^{2 n} \alpha_{i} X^{i}
$$

where $\alpha_{n}=1$. For each $i \neq n$, there exists a monic polynomial $h_{i}$ in $R[X]$ such that $h_{i}\left(\alpha_{i}\right)=0$; we may decompose all polynomials $h_{i}(X)$ into linear factors over some integral extension $D$ of $R$ containing $R^{\prime}$ :

$$
h_{i}(X)=\prod_{j=1}^{m_{i}}\left(X-\beta_{i j}\right) .
$$

Let $\varphi(X)$ be the product of all possible polynomials $\sum_{i=0}^{2 n} \beta_{i j_{i}} X^{i}$, where $0 \leq j_{i} \leq m_{i}$ for $i \neq n$, and $j_{n}=0, \beta_{n, 0}=1$. The coefficients of the polynomial $\varphi(X)$ can be 
expressed as polynomials in the elements $\beta_{i j}$ that are symmetric in each sequence of indeterminates $X_{i 1}, \ldots, X_{i m_{i}}$ for $i \neq n$. Thus all the coefficients of $\varphi(X)$ are in $R$. Moreover, $\varphi$ is a product of polynomials in $D[X]$ with unit content in $D$. Since $D$ is integral over $R$, the polynomial $\varphi$ has unit content also in $R$. We have $\varphi=f \psi$ for some polynomial $\psi$ over $K$. Since the polynomial $f$ is Gaussian over $R$, we obtain $R=\mathfrak{c}_{R}(\varphi)=\mathfrak{c}_{R}(f) \mathfrak{c}_{R}(\psi)$; thus $\mathfrak{c}_{R}(f)$ is an invertible ideal in $R$.

Theorem 4 implies that the Gaussian property of a polynomial over an integral domain depends just on its content. In the next corollary we present further immediate consequences of Theorem 4 .

Corollary 5. Let $R$ be an integral domain. We have

(1) If $f$ and $g$ are polynomials in $R[X]$ with the same content, then $f$ is Gaussian iff $g$ is Gaussian. In particular, a polynomial obtained from a Gaussian polynomial over $R$ by permuting its coefficients is Gaussian.

(2) A Gaussian polynomial over $R$ is Gaussian over any ring extension of $R$.

(3) All polynomials over $R$ are Gaussian iff $R$ is a Prüfer domain (see [1] Theorem 1.3]).

Example 6 (cf. Corollary 5 (2)). We consider the ring $R=k[s, t] /(s, t)^{2}$, where $k$ is a field, and $s$ and $t$ are independent indeterminates over $k$; thus all polynomials over $R$ are Gaussian (cf. [1 7]). However, if $u$ and $v$ are indeterminates over $R$, then the polynomial $s+t X$ is not Gaussian over the ring extension $R[u, v]$. Indeed, $s v \in \mathfrak{c}_{R[u, v]}(s+t X) \mathfrak{c}_{R[u, v]}(u+v X)$, but $s v \notin \mathfrak{c}_{R[u, v]}((s+t X)(u+v X))$, that is, $s v \notin(s u, t v, s v+t u)$.

Proposition 7. A factor of a nonzero Gaussian polynomial over an integral domain is not necessarily Gaussian. Moreover, if $R$ is an integral domain, $f(X) \in R[X]$, and $f^{2}$ is Gaussian, then $f$ is not necessarily Gaussian.

Proof. Indeed, let $k$ be a field of characteristic 2 , and let $R=k\left[s, t, \frac{s^{2}}{t^{2}}\right]$, where $s$ and $t$ are indeterminates over $k$. Let $f(X)=s+t X$. We have $f^{2}=s^{2}+t^{2} X^{2}$ is Gaussian since its content is generated by one element, namely, by $t^{2}$, but $f$ is not Gaussian since $s t \in(\mathfrak{c}(f))^{2} \backslash \mathfrak{c}\left(f^{2}\right)$.

However, a factor of a nonzero Gaussian polynomial over an integrally closed domain is Gaussian (a domain $R$ is integrally closed iff the multiplicative set of the polynomials with invertible content, that is, the set of nonzero Gaussian polynomials, is saturated).

We conjecture that Theorem 4 can be extended to reduced rings, to the effect that a polynomial over a reduced ring is Gaussian iff its content is locally principal. However, if $R=k[s, t] /(s t)$, where $k$ is a field, then the polynomial $f(X)=\bar{s}+\bar{t} X \in$ $R[X]$ is not Gaussian, although $R$ is a principal ideal domain modulo each of its two nonzero minimal primes, namely, $(\bar{s})$ and $(\bar{t})$. To show that the polynomial $f(X)$ is not Gaussian, let $g(X)=\bar{t}+\bar{s} X$; thus $f g=\left(\bar{s}^{2}+\bar{t}^{2}\right) X$ and we have $\bar{s}^{2} \in \mathfrak{c}(f) \mathfrak{c}(g)$, but $\bar{s}^{2} \notin \mathfrak{c}(f g)$ since $s^{2} \notin\left(s t, s^{2}+t^{2}\right) k[s, t]$ (cf. [7]).

Finally, we conjecture that Theorem 4 can be generalized to any number of indeterminates over any reduced ring $R$; it is enough to consider the case of a finite number of indeterminates. By the above proof, the conjecture holds for an integral domain $R$ of finite characteristic; more generally, it is enough to assume that the residue fields $R / M$ for $M$ a maximal ideal in $R$, are of finite characteristic (see [7]). 


\section{REFERENCES}

[1] D. D. Anderson and B. G. Kang, Content Formulas for Polynomials and Power Series and Complete Integral Closure, J. Algebra 181 (1996), 82-94. MR.1382027 (97c:13014)

[2] W. Bruns and A. Guerrieri, The Dedekind-Mertens formula and determinantal rings, Proc. Amer. Math. Soc. 127 (1999), 657-663. MR,1468185 (99f:13013)

[3] A. Corso, W. Heinzer, and C. Huneke, A generalized Dedekind-Mertens lemma and its converse, Trans. Amer. Math. Soc. 350 (1998), 5095-5109. MR1473435|(99b:13012)

[4] A. Corso, W. Vasconcelos, and R. Villarreal, Generic Gaussian ideals, J. Pure Appl. Algebra 125 (1998), 117-127. MF 1600012 (98m:13014)

[5] P. Eakin and A. Sathaye, Prestable ideals, J. Algebra 41 (1976), 439-454. MR0419428 $(54: 7449)$

[6] R. Gilmer, Multiplicative Ideal Theory, Queen's Papers in Pure and Applied Mathematics, vol. 90 1992, Kingston, Ontario, Canada. MR1204267 (93j:13001)

[7] S. Glaz and W. V. Vasconcelos, The Content of Gaussian Polynomials, J. Algebra 202 (1998), 1-9. MR,1614237 (99c:13003)

[8] W. Heinzer and C. Huneke, Gaussian polynomials and content ideals, Proc. Amer. Math. Soc. 125 (1997), 739-745. MR 1401742 (97e:13015)

[9] W. Heinzer and C. Huneke, The Dedekind-Mertens Lemma and the contents of polynomials, Proc. Amer. Math. Soc. 126 (1998), 1305-1309. MR.1425124 (98j:13003)

Department of Mathematics, Ohio State University-Newark, Newark, Ohio 43055

E-mail address: lopera@math.ohio-state.edu

Department of Mathematics, University of Haifa, Haifa 31905, Israel

E-mail address: mroitman@math.haifa.ac.il 